López-Mayor, C. \& Cascales-Martínez, A. (2019). Acción tutorial y tecnología: propuesta formativa en educación primaria. Revista Electrónica Interuniversitaria de Formación del Profesorado, 22(3), 233-249.

DOI: http://dx.doi.org/10.6018/reifop.22.3.347231

\title{
Acción tutorial y tecnología: propuesta formativa en educación primaria
}

Cristian López Mayor, Antonia Cascales-Martínez

Universidad de Murcia

\section{Resumen}

La mayoría de los menores de edad hace uso diario de las tecnologías, por lo que abordar las competencias digitales desde la acción tutorial a través de la función orientadora de todos los docentes es fundamental. El objetivo es describir el conocimiento inicial de los docentes sobre el plan de acción tutorial, temáticas y las competencias digitales en acción tutorial. Para ello se ha diseñado e implementado una acción formativa ad hoc de forma telemática, en acción tutorial y tecnologías. Siendo la muestra 26 tutores de Educación Infantil y Primaria. Para la recogida de datos se diseñaron dos cuestionarios y una encuesta de satisfacción. Los resultados ponen de manifiesto que hay una actitud favorable hacia las competencias digitales y su inclusión en la acción tutorial, esta actividad formativa mejoró la competencia tecnológica dentro de la acción tutorial.

\section{Palabras clave}

Acción tutorial; competencia digital; formación de docentes; aprendizaje en línea.

\section{Tutorial Action and Technology: Formative Proposal in Primary Education}

\begin{abstract}
The vast majority of under-age people use technology daily. We believe that it is essential to work on these topics and mastering digital competences in tutorial actions through the guiding function of all teachers. The current investigation describes the initial knowledge of teachers on topics related to the tutorial action plan and the digital competences in tutorial action. The study sample was 26 participants of pre-school and primary education teachers. The tools used to collect information were two questionnaires and a Satisfaction Survey. The results of the analysis show that there is a favourable attitude towards digital competences and their inclusion in the tutorial action, this training course led to a slight improvement in the competences in technology and tutorial action.
\end{abstract}




\section{Key words}

Tutorial action; digital competences; teacher training; e-learning.

\section{Introducción}

En el terreno educativo, la acción tutorial desarrollada en centros escolares posee un papel crucial para el desarrollo académico, personal y social del alumnado según la Ley Orgánica 2/2006, de 3 de mayo, de Educación (LOE, 2006). Dentro de la orientación educativa, la acción tutorial, constituye un proceso que complementa la acción docente a fin de atender a la diversidad del alumnado (Morales, 2010).La acción tutorial se desarrolla de forma colaborativa entre familiares, profesorado y alumnado para conseguir el desarrollo de los fines educativos que se deben trabajar en el centro educativo. Y, por tanto, la tutoría es inherente a la función docente en la medida que debe implicar a todo el profesorado en la labor orientadora (Del Pozo, 2011).

Los docentes cooperan en la adecuada orientación de sus alumnos, en la detección de necesidades educativas, en la propuesta y aplicación de medidas, en la evaluación de resultados, así como en la oportuna información a alumnos y familias, para conseguir una comunicación fluida y eficaz. Por otro lado, la tutoría es la acción tutorial que específicamente realiza el tutor (entrevistas con las familias, sesiones de tutoría, etc.), la labor orientadora que realiza el tutor, de la cual él es la persona responsable de su coordinación (Álvarez \& Bisquerra, 2012).

Podemos concluir definiendo la acción tutorial como un proceso orientador intencional y planificado llevado a cabo por el profesorado, en el ejercicio de su función docente, realizando una labor de acompañamiento continuo y personalizado a cada alumno y grupo que garantice el desarrollo integral en todos los ámbitos (académico, social, personal y profesional).

Al margen de las acciones, actividades y programas utilizados en la acción tutorial, de carácter más tradicional (Álvarez \& Bisquerra, 2012; Del Pozo, 2011; Pacheco et al., 2006; Royo et al., 2002), consideramos la inclusión de otras acciones en relación a las competencias digitales que, creemos, trabajan la acción tutorial en sus principios, objetivos y ámbitos de actuación. Actividades como, entre otras, aprender las normas de un uso adecuado de los dispositivos electrónicos (Enseñar a ser), trabajar y formar a las familias en tecnologías de la información, para que sus hijos tengan normas y hagan un uso adecuado (Enseñar a hacer), los roles en situaciones de acoso (Enseñar a vivir juntos), también en la red y en una perspectiva más amplia. Además, actividades para trabajar la conexión fundamental entre docentes y alumnado, a través de la tecnología, mediante el uso de aplicaciones educativas (Enseñar a vivir juntos).

Siguiendo la línea argumentativa del presente trabajo, sabemos que la acción tutorial es una labor de todo el profesorado y también que la calidad de la enseñanza y del profesorado son factores clave para la calidad educativa. Por lo tanto, podemos decir que la formación del profesorado es decisiva para la calidad educativa (Escudero, González \& Rodríguez, 2018). Y en dicha formación, estudios recientes alertan de la necesidad de formación para el profesorado en competencias digitales, en el cambio de actitudes hacia el uso de dichas tecnologías en la escuela y sus transferencias a las aulas con rigor pedagógico (Marciá \& Garreta, 2018; Pérez \& Rodríguez, 2016).

En los últimos años se están produciendo grandes cambios y a gran velocidad en cuanto a la profesión docente. Con la inclusión de las tecnologías en los centros educativos y la función socializadora que se le requieren a los docentes en la actualidad, es de vital importancia, 
buscar alternativas y nuevos cauces para hacer frente a los nuevos retos sociales emergentes y que los docentes tengan un desarrollo profesional.

Entendemos por desarrollo profesional docente, a toda actividad enfocada a la mejora de las competencias docentes actuales, es decir, toda actividad enfocada a la mejora de la calidad docente (Tello \& Aguaded, 2009). Desde este punto de vista la formación permanente es de vital importancia. Esta formación continua tiene su base en la constante y rápida evolución que experimenta nuestra sociedad, y por tanto, la adecuación de los sistemas educativos y métodos de enseñanza a esta. Si para cualquier docente la formación continua es crucial en su desarrollo profesional, para el tutor, entendiendo que es el guía, mediador, acompañante del alumno en su desarrollo personal, social, emocional e integral, los es más aún si cabe.

La tendencia actual de la formación permanente es hacia la integración de las tecnologías en la Educación, promueven la importancia de la formación del profesorado en el desarrollo de la competencia digital. César Bernal y Antonia Rodríguez (2007) afirman que las propuestas e-learning adaptadas a las necesidades del profesorado, son muy útiles como recurso pedagógico de formación permanente del profesorado.

Las tecnologías, además, se han convertido en la vía más utilizada en la formación permanente del profesorado. Señala David Gordon (2003), a través de espacios formales de formación online el docente tiene una oportunidad de formación flexible, conveniente y sostenida, que sus predecesores nunca tuvieron. Además les permite publicar digital e impresamente las experiencias docentes que lleven a cabo (Carneiro, Toscano \& Díaz, 2011), dando lugar a una interacción con otros docentes o investigadores a nivel mundial (ArufeGiráldez, Soidán, Furelos \& Patón, 2016).

La formación en línea o e-learning es un proceso formativo, que está orientado a la adquisición de una serie de competencias y destrezas en un contexto social y que se desarrolla en un ecosistema tecnológico en el que interactúan diferentes perfiles de usuarios, los cuales, comparten contenidos, actividades y experiencias y que además, en situaciones de aprendizaje formal, debe ser tutelado por actores docentes cuya actividad contribuya a garantizar la calidad de todos los factores involucrados(García-Peñalvo \& Seoane Pardo, 2015). Suele implementarse a través de estos ecosistemas tecnológicos. Entre las herramientas más utilizadas están los Sistemas de Gestión de Aprendizaje o LMS (Learning Management System). Estos ecosistemas tecnológicos cuentan con módulos administrativos que permiten, entre otras cosas, configurar cursos, matricular alumnos, registrar profesores, y asignar calificaciones.

Las plataformas ofrecen paquetes de herramientas vinculadas a diferentes dimensiones comunicacionales, como correo electrónico, chat, foros, wikis y bases de datos, sobre las cuales pueden desplegarse diferentes tipos de actividades, tanto grupales como individuales (Marín\& Llorente, 2015; Marín, Ramírez\& Sampedro, 2011).En la actualidad, la interacción social constituye una de las principales actividades de los usuarios de Internet. Aparecen conceptos como comunidad de comunidades (Torres \& Gago, 2014), que parten de la perspectiva de que los participantes en un MOOC (Masive Online Open Course, cursos en línea masivos y abiertos) se convierten en miembros de la comunidad que aportan su conocimiento para que otros aprendan; en esa Comunidad de Comunidades, señalan, estas pueden ser no solo de aprendizaje, sino también de carácter práctico, donde los participantes interactúan para crear y emprender juntos. Francisco José García-Peñalvo y Antonio Miguel Seoane (2015) se refieren a comunidades en el medio digital indicando su gran valor dentro del ámbito educativo y reafirmándose en lo que se apuntaba una década antes. Desde nuestro punto de vista, una de las estrategias que debe desarrollarse con la teleformación es 
el aprendizaje colaborativo y cooperativo, y ello pasa inicialmente por la potenciación de la creación de un sentimiento de comunidad entre los diferentes participantes, que será al mismo tiempo la base de una comunidad virtual.

En la Tabla 1, establecemos las competencias digitales del docente para trabajar en los tres ejes de desarrollo de la acción tutorial y actividades concretas en las que las tecnologías tengan un papel importante.

Tabla 1.

Competencias digitales y Acciones Tutoriales mediante el uso de las tecnologías en los distintos ámbitos

\begin{tabular}{|c|c|c|}
\hline Ámbito & $\begin{array}{l}\text { Competencias } \\
\text { digitales }\end{array}$ & Actividades \\
\hline $\begin{array}{ll}\text { Con los } & \text { los } \\
\text { alumnos } & \end{array}$ & 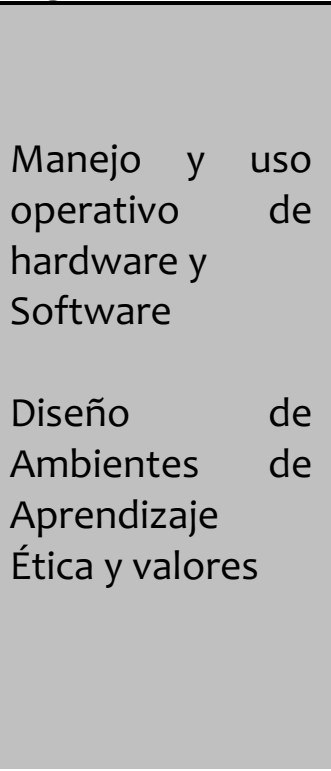 & $\begin{array}{l}\text { Aplicaciones, buscadores educativos y } \\
\text { plataformas educativas. } \\
\text { Diseño de actividades con las familias y los } \\
\text { estudiantes, se pueden crear también con } \\
\text { respecto a los contenidos de la acción tutorial, } \\
\text { como pueden ser las normas, tanto las de clase } \\
\text { como en este caso de uso adecuado de las } \\
\text { tecnologías. } \\
\text { Ambientes de aprendizaje virtuales destinados } \\
\text { a los alumnos. } \\
\text { Se trabajan el respeto a los compañeros tanto } \\
\text { en el ambiente educativo como en el virtual, se } \\
\text { transmiten valores, que son extensibles al } \\
\text { ambiente virtual, la ciberresponsabilidad, } \\
\text { prevención de ciberacoso escolar. }\end{array}$ \\
\hline \multirow{3}{*}{$\begin{array}{l}\text { Con el } \\
\text { profesorado }\end{array}$} & $\begin{array}{l}\text { Vinculación TIC } \\
\text { con el Currículo }\end{array}$ & $\begin{array}{l}\text { Se trabajan en Aplicaciones educativas y } \\
\text { plataformas para trabajar el currículum. } \\
\text { Actividades que usan aplicaciones educativas } \\
\text { para trabajar los contenidos establecidos en el } \\
\text { currículum. }\end{array}$ \\
\hline & $\begin{array}{lr}\text { Evaluación } & \text { de } \\
\text { recursos } & y \\
\text { Aprendizaje } & \end{array}$ & $\begin{array}{l}\text { Se plantean sistemas alternativos de evaluación } \\
\text { a través de tecnologías. } \\
\text { Capacidad de elaborar cuestionarios virtuales, } \\
\text { encuestas, evaluación a través de juegos. }\end{array}$ \\
\hline & $\begin{array}{l}\text { Mejoramiento } \\
\text { profesional }\end{array}$ & $\begin{array}{l}\text { Compartir recursos. } \\
\text { Se llevan a cabo acciones formativas o } \\
\text { comunidades de aprendizaje que implementen } \\
\text { las tecnologías para llevar acabo las acciones } \\
\text { tutoriales. }\end{array}$ \\
\hline $\begin{array}{l}\text { Con } \\
\text { familias }\end{array}$ & Ética y valores & $\begin{array}{l}\text { Programas de Control parental, buscadores } \\
\text { educativos y plataformas educativas. }\end{array}$ \\
\hline
\end{tabular}




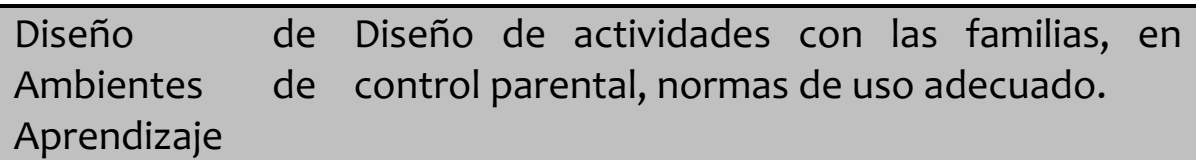

Fuente: Elaboración propia basada en Silva et al. (2006)

Por todo ello, el objetivo general de esta investigación es evaluar los conocimientos que poseen los docentes de Educación Infantil y Primaria sobre tecnologías antes de la implementación de una acción formativa como parte de la acción tutorial y, de este modo, comprobar su conocimiento en el desarrollo de la acción tutorial en cuanto a tecnologías se refiere tras recibir formación ad hoc. Este objetivo general se concreta en los específicos siguientes:

(1) Explorar el conocimiento inicial de las docentes de Educación Infantil y Educación Primaria sobre el Plan de Acción Tutorial, temáticas y sus competencias digitales en acción tutorial.

(2) Diseñar e implementar una Acción formativa con los docentes de Infantil y Primaria en tecnologías de la información y acción tutorial.

(3) Analizar si han existido cambios en las competencias de los docentes tras la acción formativa.

\section{Metodología}

El diseño adoptado ha sido de corte descriptivo, con diseño de intervención pretest-postest de grupo único, desarrollado a través de una acción telemática. Para la evaluación de la acción formativa se diseña ad hoc y aplica un cuestionario en la fase inicial y se vuelve a aplicar en la fase final, una vez cursada la acción formativa, aplicando asimismo una escala que evalúa la satisfacción y calidad de la intervención desde la perspectiva de los docentes participantes (Mengual, Lloret \& Roig, 2015).

La población son docentes de Infantil y Primaria de la Región de Murcia. Se ha tratado de un muestreo deliberado donde la muestra invitada y aceptante ha sido un total 55 docentes en activo. Si bien la muestra es de 55 participantes iniciales, solamente 26 (47,3\% de la muestra Inicial, 8 hombres y 18 mujeres) finalizaron la acción formativa. En la Tabla 2, se muestran los datos demográficos de interés, como la edad, experiencia docente, nivel de informática, tipo de docencia y etapa educativa de los participantes.

Tabla 2

Datos demográficos de los participantes en ambas fases $(\mathrm{N}=26)$

\begin{tabular}{llll} 
& & Frecuencia & Porcentaje \\
\hline \multirow{2}{*}{ Género } & Masculino & 8 & 30,8 \\
\cline { 2 - 4 } & Femenino & 18 & 69,2 \\
\hline \multirow{3}{*}{ Conocimiento de Informática } & Básico & 2 & 7,7 \\
\cline { 2 - 4 } & Usuario & 21 & 80,8 \\
\cline { 2 - 4 } & Avanzado & 3 & 11,5 \\
\hline
\end{tabular}




\begin{tabular}{llll}
\multirow{2}{*}{ Tipo de Funcionario } & \multicolumn{1}{l}{ Funcionario } & 17 & 65,4 \\
\cline { 2 - 4 } Etapa educativa & Interino & 9 & 34,6 \\
\hline & Primaria & 23 & 88,5 \\
\hline \multirow{3}{*}{ Edad } & Infantil & 3 & 11,5 \\
\cline { 2 - 4 } & $<25$ años & 2 & 7,7 \\
\hline $25-35$ años & 14 & 53,8 \\
\hline $35-45$ años & 7 & 26,9 \\
\hline & $45-55$ años & 3 & 11,5 \\
\hline & $>55$ años & 0 & 0 \\
\hline Experiencia Docente & $<5$ años & 5 & 19,2 \\
\hline $5-12$ años & 12 & 46,2 \\
\hline $12-20$ años & 6 & 23,1 \\
\hline $20-28$ años & 2 & 7,7 \\
\hline$>28$ años & 1 & 3,8 \\
\hline
\end{tabular}

\section{Procedimiento}

A partir de la revisión bibliográfica y de programas de formación online para tutores, de manera sistemática, se procedió al diseño de la acción formativa que se detalla en el apartado de resultados, la cual fue sometida a una revisión exhaustiva. Una vez diseñada y depositada en la plataforma Google Clasroom, se llevó a cabo una presentación informativa sobre el curso o acción formativa al profesorado de cada centro. El contenido de la misma era una introducción a esta acción formativa en línea. Se les transmitió la duración de la misma ( 5 horas), las herramientas necesarias (Ordenador o sucedáneos y conexión a internet), y el modo de acceso a la plataforma, LMS, utilizada para llevar a cabo la acción formativa.

En segundo lugar, se administró, de manera telemática, el Cuestionario de Evaluación Inicial y se animó a la inscripción, para ello se diseñó un cartel que se colocó en los centros educativos visitados a modo de estimulador para inscribirse en la acción formativa de forma voluntaria, en el curso: Tecnologías en mi aula y en casa. Plan de Acción Tutorial.

En tercer lugar, se procedió a la realización de la acción formativa que consistía en 3 módulos de una duración de 2 horas el módulo uno y de 1 hora cada uno de los dos módulos restantes. Teniendo en cuenta las características de la formación telemática (Gil \& Martínez, 2017), el diseño de la acción fue predominantemente activo, enfocada a la realización de actividades que implican: lectura o visionado de los materiales suministrados, cumplimentar las tareas de carácter práctico y reflexivo y la comunicación bidireccional mediante el foro, los mensajes en el tablón y el correo interno. El contenido y el desarrollo de la acción formativa creemos conveniente desarrollarlos en la sección de resultados, de acuerdo al objetivo segundo de este trabajo.

Para finalizar, una vez cumplimentada la acción formativa, se les planteó el cuestionario de Evaluación Final y la Escala de Satisfacción del curso de manera telemática. Una vez recogidos todos los datos, se procedió al análisis de resultados, al establecimiento de conclusiones y a la redacción del informe final de investigación. 


\section{Instrumentos}

Para la recogida de datos de los participantes se utilizó el método de encuesta elaboradas ad hoc para la presente investigación en base a los objetivos de la investigación. En la primera escala se recogió la percepción sobre el conocimiento que tienen los participantes en relación al plan de acción tutorial (PAT), las temáticas tratadas en el PAT y algunas competencias digitales docentes (Roblizo \& Cózar,2015; Silva et al., 2006). La segunda escala es una escala de Satisfacción sobre la acción formativa.

A continuación se describen brevemente los dos instrumentos diseñados y aplicados para la recogida de información.

\section{Cuestionario de Evaluación Inicial y Evaluación Final}

En ella se recogieron, por un lado, los datos demográficos (Edad, Experiencia Docente, Etapa Educativa, Género, Tipo de funcionariado y Conocimiento de Informática), y por otro, la percepción sobre el conocimiento que tienen los participantes en relación al plan de acción tutorial, las temáticas tratadas en el PAT y las competencias digitales. La escala contiene 17 ítems tipo Likert de respuesta sobre una serie de afirmaciones que varían de 1 a 5 puntos, donde 1 es "Nada de acuerdo" y 5 es "Totalmente de acuerdo". La escala tiene una fiabilidad con una consistencia interna buena (Alfa de Cronbach $=0,856$ ) y validez de contenido (interjueces), y está dividida en 3 dimensiones: Conocimiento del PAT, temáticas de la acción tutorial, las competencias tecnológicas y acción tutorial, tal y como se puede apreciar en la Tabla 3.

Tabla 3

Dimensiones integradas en el Cuestionario $N^{\circ} 1$ Evaluación Inicial y Final

\section{Evaluación \\ Inicial y}

\section{Evaluación}

Final

Dimensiones

Ítems Ejemplo de ítem

"Los docentes pueden proponer contenidos a trabajar en la acción

Conocimiento del PAT $1,2,3$ tutorial"

"4. Considero interesante tratar la temática del conocimiento de grupo-

Temáticas de la acción 4, 5, 6, clase como contenido de la acción tutorial $8, \quad$ tutorial."

Cuestionario de 17 Ítems tipo Likert (puntuación de 1 hasta 5)
"9. Considero adecuado tratar con los estudiantes de Educación Infantil y

7, 9, Primaria el uso de normas de seguridad Competencia Digital y 10, 11, en Internet o del uso adecuado de acción tutorial 12,13, dispositivos móviles."

Ítems referidos a la formación de familias y tutores sobre 14,15,1 “17. Necesito formación permanente sobre estas temáticas."

tecnología $\quad 6,17$




\section{Encuesta de Satisfacción}

Esta escala muestra la satisfacción con respecto a la acción formativa. Se ha elaborado en base a unos criterios de calidad, una adaptación del Cuestionario de evaluación de la calidad virtuales adaptados a MOOC de Santiago Mengual et al. (2015) y una fiabilidad alta, según el Alfa de Cronbach del 0,797.Consta de 5 ítems tipo Likert de respuesta sobre una serie de afirmaciones que varían de 1 a 5 puntos, donde 1 es "Nada de acuerdo" y 5 es "Totalmente de acuerdo". Y una pregunta de tipo abierta sobre las posibilidades de mejora de la calidad del curso.

\section{Análisis de datos}

Conforme a los objetivos de la investigación y la naturaleza de los datos recabados se realizó una aproximación de tipo cuantitativo. Para el análisis de los datos se usó el paquete estadístico SPSS en su versión 20.0, se realizó un estudio descriptivo de cada pregunta del cuestionario de Evaluación Inicial y Final, se analizó por un lado de manera descriptiva y por otro el efecto de la acción formativa de los participantes que finalizaron la acción formativa, a través de técnicas de contraste de muestras relacionadas. Tras comprobar los contrastes en los que se apreciaban diferencias estadísticamente significativas, se procedió a calcular el tamaño del efecto por medio del estadístico "d" de Cohen (1988), quien establece que dicho valor ha de ser igual o superior a 0,5 para considerar un tamaño del efecto adecuado.

\section{Resultados}

Presentamos los resultados por objetivos, en primer lugar describiremos el conocimiento inicial de los docentes, en segundo lugar, se describirá la acción formativa, su diseño y su implementación y por último, se evalúan y analizan los cambios en las mismas competencias de los docentes tras la acción formativa.

OBJETIVO 1. Explorar el conocimiento inicial de los docentes de Infantil y Primaria sobre el Plan de Acción Tutorial, temáticas y sus competencias digitales en acción tutorial.

Se procede al análisis de los resultados de las dimensiones del cuestionario de Evaluación inicial. Se presentan los resultados de las medias en las 3 dimensiones del cuestionario. En primer lugar, según se expone en la Tabla 4, los docentes participantes en la acción formativa parten inicialmente con un Conocimiento del PAT situado en una media de 4,76 (sobre un máximo de 5), destacando su consideración del PAT como un documento básico de consulta para el docente $(M=4,92)$.

Tabla 4

Conocimiento que tienen los docentes del Plan de Acción Tutorial. Medias y desviaciones típicas

\begin{tabular}{lll} 
Ítems & Media & Desviación Típica \\
\hline $\begin{array}{l}\text { El Plan de Acción Tutorial es un documento que el } \\
\text { maestro ha de consultar. }\end{array}$ & 4,92 &, 272 \\
\hline $\begin{array}{l}\text { Los contenidos a trabajar en la acción tutorial deben } \\
\text { aparecer en el PAT. }\end{array}$ & 4,81 &, 402 \\
\hline
\end{tabular}


Los docentes pueden proponer contenidos a trabajar en la acción tutorial.

$4,58 \quad, 578$

Dimensión Conocimiento del PAT.

4,76

,294

En cuanto a la segunda dimensión, en relación al interés que muestran en incluir diversas Temáticas en el PAT, los resultados quedan recogidos en la Tabla 5. Todas las puntuaciones están próximas a la puntuación máxima de 5(Totalmente de Acuerdo), es decir, todas las temáticas propuestas resultan de gran interés por los docentes participantes para ser incluidas en su acción tutorial.

\section{Tabla 5}

Percepción del interés que tienen los docentes en incluir temáticas dentro del Plan de Acción Tutorial. Medias y desviaciones típicas

\begin{tabular}{lll} 
Ítems & Media & $\begin{array}{c}\text { Desviación } \\
\text { Típica }\end{array}$ \\
\hline $\begin{array}{l}\text { Considero interesante tratar la temática del conocimiento } \\
\text { de grupo-clase como contenido de la acción tutorial. }\end{array}$ & 4,88 &, 326 \\
\hline $\begin{array}{l}\text { Considero interesante tratar actitudes y hábitos de estudio } \\
\text { como contenido de la acción tutorial. }\end{array}$ & 4,77 &, 430 \\
\hline $\begin{array}{l}\text { Considero interesante tratar la Educación Emocional como } \\
\text { contenido de la acción tutorial. }\end{array}$ & 4,88 &, 326 \\
\hline $\begin{array}{l}\text { Considero interesante tratar la educación en valores como } \\
\text { contenido de la acción tutorial. }\end{array}$ & 4,96 &, 196 \\
\hline $\begin{array}{l}\text { Dimensión temáticas del PAT } \\
\text { 4. 203 }\end{array}$ & 4,87 &, 203 \\
\hline
\end{tabular}

Los resultados de la tercera dimensión, competencias digitales en cuanto a la acción tutorial, pueden verse en la Tabla 6 . Los docentes participantes, en el momento inicial, se muestran entre De acuerdo y Totalmente de acuerdo en incluir las temáticas referidas al uso seguro y educativo de las TIC en la acción tutorial.

Tabla 6

Percepción de los docentes sobre la importancia de trabajar las competencias digitales en cuanto a la acción tutorial. Medias y desviaciones típicas

Ítems

Media

Desviación Típica

Considero interesante tratar el uso adecuado de dispositivos móviles (móvil, Tablet,...) e Internet como contenido de la acción tutorial.

$4,50 \quad 648$

Considero adecuado tratar con los alumnos de Educación Infantil y

Primaria el uso de normas de seguridad en Internet o del uso

$4,27 \quad 604$ 
adecuado de dispositivos móviles como contenido de la acción tutorial.

Considero necesario trabajar en el aula las normas de utilización de redes sociales, buscadores, plataformas de imágenes o vídeos 4,27 ,778 (Youtube, Google,...) como contenido de la acción tutorial.

Es adecuado proponer a los estudiantes de Infantil y Primaria que realicen búsquedas en Internet.

$4,15 \quad, 834$

Creo que es necesario abordar en las etapas de Infantil y Primaria la temática del ciberacoso, en concreto el rol de víctima, el rol del $\quad 4,27 \quad, 778$ acosador, y el rol de observador participante.

Creo adecuado abordar estas temáticas en las reuniones grupales y tutorías con las familias.

Los resultados de los ítems sobre la formación de familias y docentes en tecnología quedan recogidos en la Tabla 7.

Tabla 7

Percepción que tienen los docentes sobre la formación de familias y docentes en tecnología. Medias y desviaciones típicas

\begin{tabular}{|c|c|c|}
\hline Ítems & Media & Desviación Típica \\
\hline $\begin{array}{l}\text { 14. Considero que las familias tienen necesidades de } \\
\text { formación en estas temáticas. }\end{array}$ & 4,62 & ,623 \\
\hline $\begin{array}{l}\text { 15. He recibido formación sobre plataformas, } \\
\text { aplicaciones y buscadores que se ajusten a la edad de } \\
\text { alumnos de Infantil y Primaria. }\end{array}$ & 3,96 & ,916 \\
\hline $\begin{array}{l}\text { 16. La formación inicial recibida sobre acción tutorial } \\
\text { ha sido suficiente/adecuada. }\end{array}$ & 3,50 & ,707 \\
\hline $\begin{array}{l}\text { 17. Necesito formación permanente sobre estas } \\
\text { temáticas. }\end{array}$ & 3,92 & ,845 \\
\hline
\end{tabular}

Los ítems, 15, 16 y 17, tuvieron puntuaciones cercanas a 3(Ni de acuerdo ni en desacuerdo) y 4 (De acuerdo) respectivamente. Es decir, los docentes se encuentran entre Ni de acuerdo ni en desacuerdo y De acuerdo en que la formación inicial es suficiente, en que han sido formados en competencias digitales y la necesidad de que deben formarse permanentemente en tecnologías.

OBJETIVO 2. Diseñar e Implementar una Acción formativa con los docentes de Infantil y Primaria en tecnologías de la información y acción tutorial.

Descripción de la acción formativa. 
Se trata de una acción formativa de tipo telemática, alojada en una plataforma de aprendizaje, Google Classroom, a la que se puede acceder a través de la web o a través de una aplicación móvil. En la tabla 8 está el esquema de las actividades llevadas a cabo durante la acción formativa. Desde la presente investigación es importante que en todas las fases de aplicación, diseño y desarrollo de las acciones de e-learning, los profesores tengan dos tipos de presencia: cognitiva y social.

Los objetivos del curso fueron los siguientes:

-Concienciar al profesorado de la necesidad de trabajar la acción tutorial desde las tecnologías.

-Establecer las normas para un uso responsable de los dispositivos tecnológicos del menor, tanto en relación al tiempo de su uso diario y en relación a la franja de edad del menor.

-Conocer algunas de las aplicaciones, buscadores educativos más utilizados y su uso adecuado por parte de los niños/as.

-Utilizar Internet y algunas redes sociales con una finalidad pedagógica y de forma segura.

-Conocer el concepto de ciberacoso y los distintos roles que intervienen, conociendo a su vez estrategias de prevención.

Contenidos del curso

Tabla 8

Esquema sobre las actividades de la Acción formativa realizada

\begin{tabular}{|c|c|c|c|}
\hline Módulo & Objetivo & $\begin{array}{l}\text { Pilar de la } \\
\text { Educación }\end{array}$ & Actividades \\
\hline Presentación & $\begin{array}{l}\text { Concienciar al } \\
\text { profesorado de la } \\
\text { necesidad de } \\
\text { trabajar la acción } \\
\text { tutorial desde las } \\
\text { tecnologías }\end{array}$ & $\begin{array}{l}\text { Enseñar a hacer } \\
\text { Enseñar a vivir } \\
\text { juntos } \\
\text { Enseñar a conocer }\end{array}$ & $\begin{array}{l}\text { 1. Razones sobre la relevancia } \\
\text { de la temática. } \\
\text { 2. ¿Por qué es interesante } \\
\text { trabajar las tecnologías desde } \\
\text { el PAT? } \\
\text { Apertura del foro. }\end{array}$ \\
\hline $\begin{array}{l}\text { Módulo 1. Normas } \\
\text { para usar las } \\
\text { tecnologías }\end{array}$ & $\begin{array}{l}\text { Establecer las } \\
\text { normas para un uso } \\
\text { responsable de los } \\
\text { dispositivos } \\
\text { tecnológicos del } \\
\text { menor, tanto en } \\
\text { relación al tiempo } \\
\text { de su uso diario y } \\
\text { en relación a la } \\
\text { franja de edad del } \\
\text { menor. } \\
\text { Utilizar Internet y } \\
\text { algunas redes } \\
\text { sociales con una }\end{array}$ & $\begin{array}{l}\text { Enseñar a ser } \\
\text { Enseñar a vivir } \\
\text { juntos } \\
\text { Enseñar a hacer }\end{array}$ & $\begin{array}{l}\text { 3. Los niños y las nuevas } \\
\text { tecnologías: beneficios y } \\
\text { peligros. } \\
\text { 4. Decálogo de buenas } \\
\text { prácticas con el uso de las } \\
\text { tecnologías digitales. } \\
\text { 5. Ejemplos de una actividad de } \\
\text { tutoría(Alumnos y familiares). }\end{array}$ \\
\hline
\end{tabular}


finalidad

pedagógica y de

forma segura.

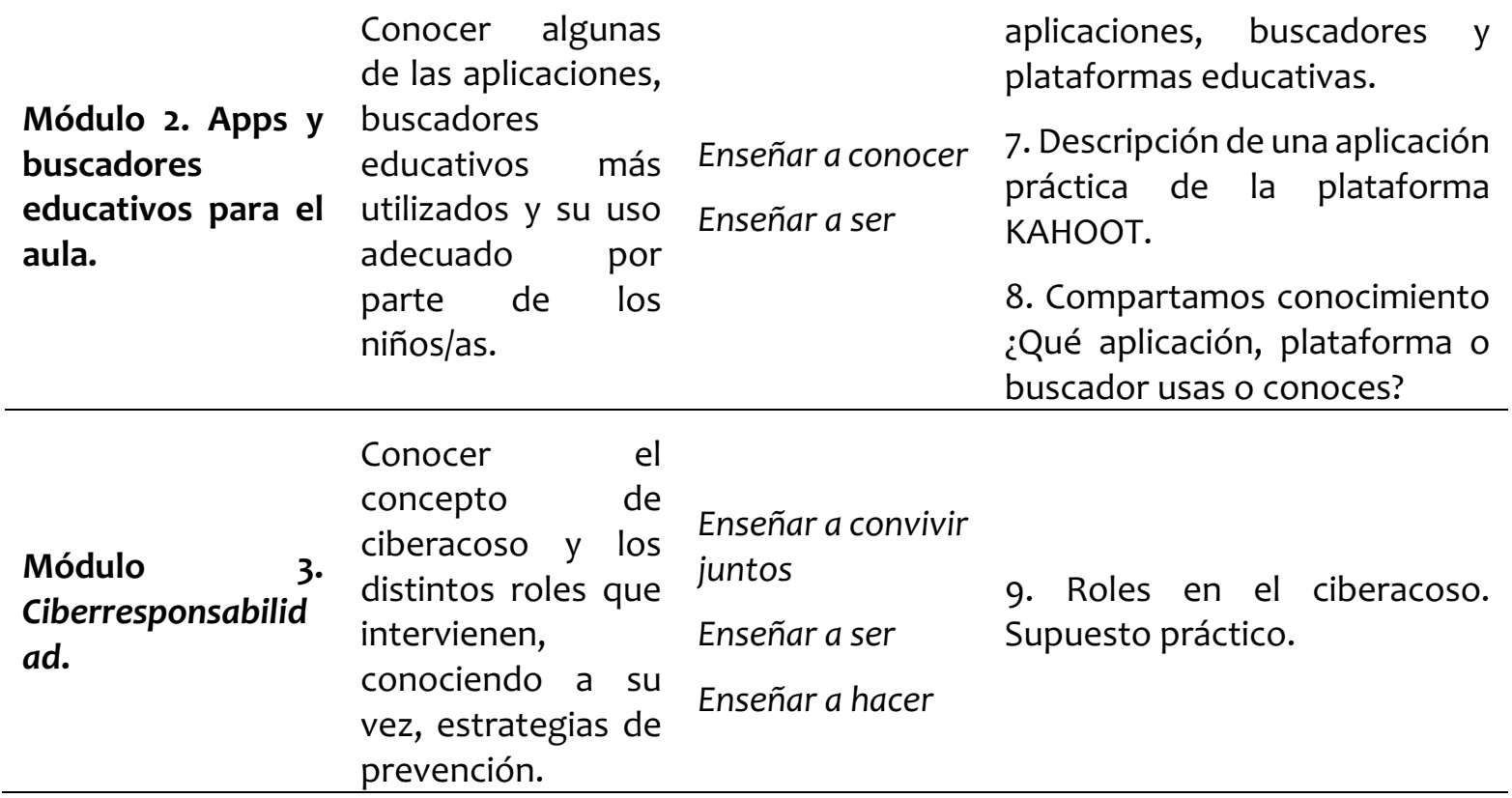

Para valorar en qué medida los docentes están satisfechos con la acción formativa realizada, se aplicó el cuestionario de satisfacción diseñado ad hoc. En la Figura 1, se muestran los resultados de la Encuesta de satisfacción del curso. En cuanto al contenido teórico y práctico del curso la media fue 4,32, la relativa a la accesibilidad y duración, 4,27, por otro lado en cuanto a la satisfacción por los recursos empleados, 4,23, en relación a la atención a los alumnos y ayuda prestadas en el curso, 4,5 y por último, la satisfacción media del curso fue de 4,41. Puntuaciones todas cercanas a 5 (Totalmente Satisfecho).

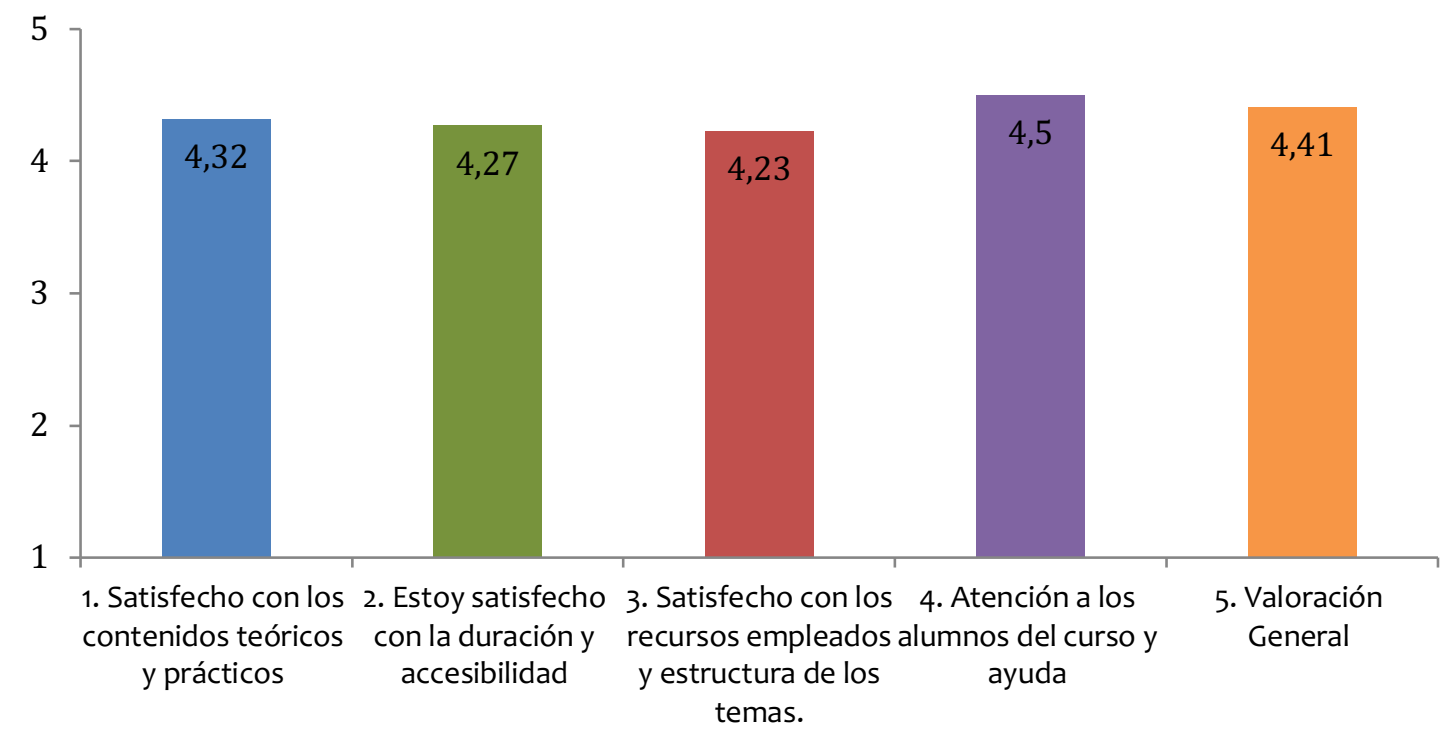

Figura 1. Puntuaciones medias de las preguntas del Cuestionario de Satisfacción. 
OBJETIVO 3. Analizar si han existido cambios en las competencias de los docentes tras la acción formativa.

A continuación se muestran las medias de las tres dimensiones de la Evaluación, tanto la final como la inicial (Ver Figura 2). Las puntuaciones en la Evaluación Final de las distintas dimensiones son ligeramente más altas, en cada una de las dimensiones, que las puntuaciones de la Evaluación Inicial.

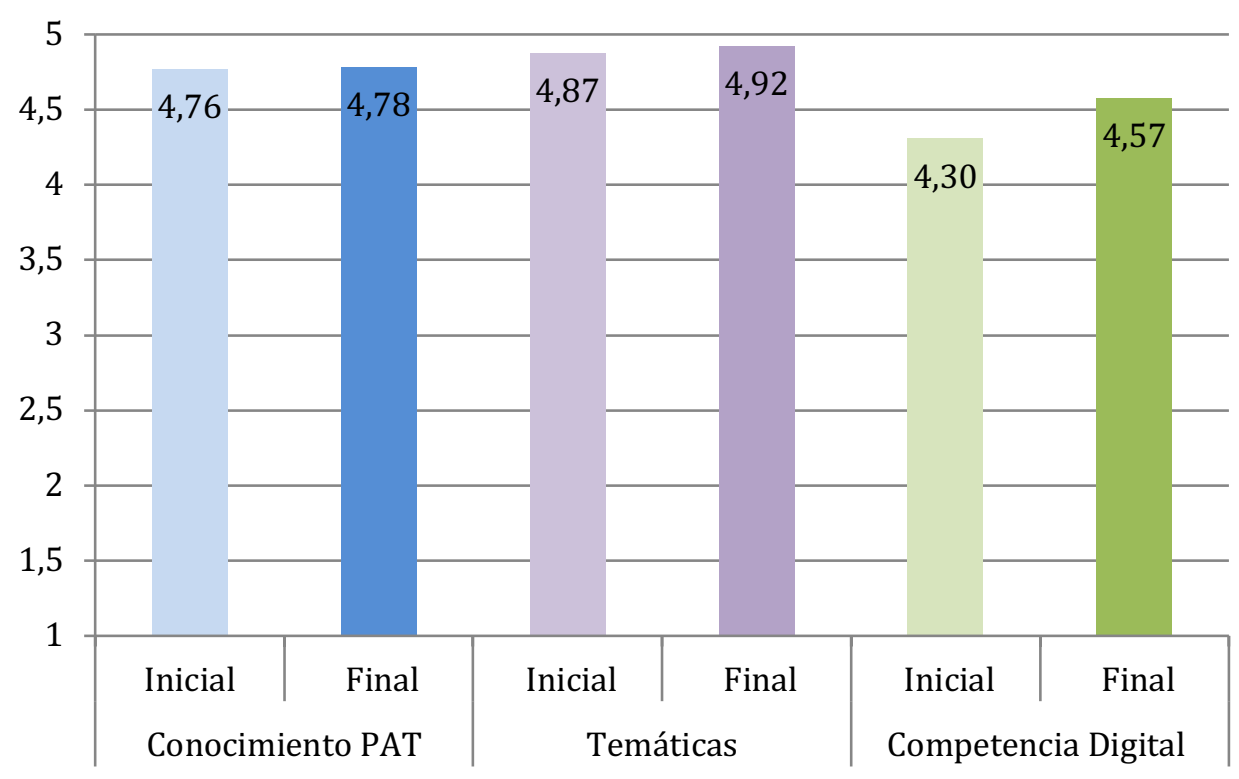

Figura 2. Medias Evaluación Inicial y Final, en base a las 3 dimensiones de la encuesta, Conocimiento PAT, Temáticas y Competencias digitales en la acción tutorial.

Para comprobar la existencia de diferencias entre el pretest y el postest, se aplicó la prueba no paramétrica para muestras relacionadas (prueba de Wilcoxon de los rangos con signo), apreciándose diferencias estadísticamente significativas $(\alpha<, 05)$ en la dimensión referida a la Competencia digital mostrada por el profesorado participante, que aumenta significativamente después de cursar la acción formativa online. Asimismo, se aprecian diferencias estadísticamente significativas en dos de los cuatro ítems referidos a la formación del docente en tecnologías, así como a su percepción sobre la necesidad de formación. Curiosamente, después de la intervención, aumenta significativamente la percepción sobre la adecuación de la formación inicial recibida, así como los docentes participantes son conscientes de una mayor necesidad de formación en estas temáticas una vez cursada la acción formativa (Tabla 9). Con respecto a la estimación del tamaño del efecto, se constata un tamaño del efecto relevante en las diferencias respecto a "Necesito formación permanente sobre estas temáticas", pues el valor d de Cohen supera el valor crítico establecido de $0,50(\mathrm{~d}=-1,102)$. El resto de contrastes no alcanzaron el valor crítico de 0,50 establecido por Cohen, aunque el contraste entre Competencias TIC inicial y final obtuvo un tamaño del efecto destacable $(\mathrm{d}=-0,496)$. 
Tabla 9

Prueba de los rangos de Wilcoxon en función del momento Pretest-Postest

\begin{tabular}{|c|c|c|c|c|}
\hline Dimensión & Momento & Media & $\mathrm{Z}$ & $\begin{array}{l}\text { Significación } \\
\text { asintótica }\end{array}$ \\
\hline \multirow[t]{2}{*}{ Competencias TIC } & Pretest- & 4,30 & \multirow{2}{*}{$-2,837$} & \multirow{2}{*}{,005 } \\
\hline & Postest & 4,57 & & \\
\hline \multirow{2}{*}{$\begin{array}{l}\text { La formación inicial recibida sobre } \\
\text { acción tutorial ha sido } \\
\text { suficiente/adecuada }\end{array}$} & Pretest- & 3,50 & \multirow{2}{*}{$-2,828$} & \multirow{2}{*}{,005 } \\
\hline & Postest & 3,81 & & \\
\hline \multirow{2}{*}{$\begin{array}{l}\text { Necesito formación permanente } \\
\text { sobre estas temáticas }\end{array}$} & Pretest- & 3,92 & \multirow{2}{*}{$-3,827$} & \multirow{2}{*}{,000 } \\
\hline & Postest & 4,73 & & \\
\hline
\end{tabular}

\section{Discusión y conclusiones}

Es crucial analizar el conocimiento de los docentes en relación a la acción tutorial y las competencias digitales relacionadas con la misma acción tutorial. En primer lugar, es destacable la muestra tan joven de la investigación, aunque de acuerdo al tipo de muestreo realizado, es congruente, ya que si no se poseen unas competencias mínimas en tecnologías, la realización de la acción formativa está condenada al fracaso (Silva et al., 2006).

Esto es coherente con los inconvenientes de este tipo de acción formativa y refleja la falta de actualización digital de los docentes más veteranos, que no han llegado a completar la acción formativa. Estos docentes entran dentro del grupo de personas que no tienen unas competencias mínimas en tecnologías. Además de la merma que supone a los alumnos para alcanzar los objetivos del currículum. Por lo tanto, desde el ámbito educativo se le tiene que dar respuesta, ya que las competencias en tecnología cada vez son más imprescindibles.

Los resultados van en la línea de las investigaciones revisadas, muestran una percepción de la importancia de las competencias digitales en todos sus ámbitos, pero es cuando se hace mención a la formación específica en tecnologías cuando los docentes obtienes resultados mediocres y su nivel de formación está por debajo del esperado (Roblizo \& Cózar, 2015). Resulta llamativo que, en cuanto a la necesidad de formación en estas competencias, no se obtengan puntuaciones tan altas en la evaluación inicial como sí ocurre con las distintas temáticas (como las normas de uso adecuado de dispositivos electrónicos, utilizar apps o plataformas educativas) en las que se centra el cuestionario.

Con respecto a la implementación de la acción formativa, esta se llevó a cabo de manera dinámica, utilizando gran variedad de recursos y medios. Se fomentó la participación y comunicación de los participantes, la reflexión y la síntesis. La acción formativa se ha diseñado e implementado teniendo en consideración los tres destinatarios de la acción tutorial: profesorado, alumnado y familias. En relación a los docentes participantes, la propia acción formativa sirve de ejemplo de ello. Entre las aportaciones del curso se ha proporcionado material, una vía abierta de comunicación entre profesionales y además encontramos que ha servido como un nuevo ambiente de aprendizaje que les ha llevado a vincular las competencias digitales con el currículum (Silva et al., 2006). En relación a la familia, se han trabajado en programas de control parental, en actuaciones tutoriales de formación de familias en materia de Normas, Aplicaciones, plataformas educativas 
adecuadas y ciberresponsabilidad. Por último, en relación a los estudiantes, se trabajó también aplicaciones, buscadores educativos, en el respeto y tolerancia etc.

La satisfacción de los docentes participantes con el curso fue bastante alta en todos los ámbitos. Se tuvo en cuenta la vertiente social, característica de las comunidades de aprendizaje, también por parte de profesor tutor ya que, el nivel y tipo de interacción entre estudiantes y profesores juega un papel fundamental en la calidad del aprendizaje y del curso(Solano, Gabriela\& Espinoza, 2017).En cuanto a las propuestas de mejora recogidas, se concretan bien en la posibilidad de premiar este tipo de acciones formativas en base a incentivos ("Que cuente como créditos de formación docente el curso") y por otro lado en la continuación de la acción formativa en una comunidad de aprendizaje poniendo en contacto a los docentes de la zona, para comunicarse y compartir recursos y experiencias, contribuyendo a la creación de redes.

Podemos concluir que los docentes participantes mejoraron significativamente en las competencias digitales en relación a su acción tutorial. Además, aumentaron significativamente su percepción sobre la adecuación de la formación inicial recibida, así como una vez realizada la acción formativa, los docentes participantes se muestran más conscientes de su mayor necesidad de formación en las temáticas cursadas. Es decir, que tras la acción formativa, los docentes muestran un significativo mayor grado de acuerdo sobre la importancia de formarse, y además de manera continua, para adquirir competencias digitales.

En cuanto a las limitaciones, la elaboración de los ítems en la escala de evaluación inicial y final podría ir encaminada a las actuaciones y conocimientos que se han realizado o se dominan y no tanto a la importancia de que se realice lo que en un ítem se expone, es decir, no tanto a la voluntad de querer trabajar en una línea sino a las temáticas desarrolladas en la acción tutorial, en su propia acción docente. Además, dado que, el tamaño de la muestra es reducido y la posibilidad de disponer de instrumentos de evaluación más precisos hacen necesario seguir indagando en las cuestiones que se plantea esta investigación. La muerte muestral elevada es frecuente en este tipo de investigación, a través de acciones formativas telemáticas, ya que según Lloyd Armstrong (2014), solo un 4\% de las personas que se inscriben en la plataforma digital Coursera, consigue completarlo. Podemos afirmar que nuestra muestra real participante que ha completado la acción formativa supera con creces este porcentaje ( 26 docentes frente a 55 que lo iniciaron, casi la mitad de éstos). Un factor clave en este éxito de participación puede residir en la campaña de sensibilización e información realizada antes en los centros educativos y el formato de duración breve del curso, no ser de larga duración.

Concluimos que la presente investigación sirve para abrir el camino a la hora de la formación en acción tutorial con competencias digitales. Han surgido interrogantes en cuanto a la necesidad de formación en tecnologías para llevar a cabo una acción tutorial de calidad y de actualidad. Este tipo de formación supone la apertura de un nuevo canal entre los docentes y la acción tutorial a través de cursos elaborados en base a las necesidades específicas de estos. Estas acciones formativas tienen que ir enfocadas a su vertiente más social, de interacción y comunicación, como comunidades de aprendizaje. Además, todavía persiste esa actitud negativa en algunos docentes que no tienen una formación mínima en tecnologías, por lo que, la comunidad educativa y la administración educativa especialmente, se debe plantear qué hacer al respecto, ya que estas competencias son cada vez más imprescindibles y necesarias.

Esta investigación tiene potenciales implicaciones para las prácticas pedagógicas relacionadas con la acción tutorial y la competencia digital. Sus resultados podrían motivar a 
profesores y centros educativos a incorporar como modalidad de formación para los docentes cursos on-line diseñados por el propio centro a fin de dotar al profesorado de los recursos necesarios para afrontar esta tarea o bien a mejorar su competencia digital, para favorecer la motivación por dar una respuesta adecuada tanto a familias como alumnado, especialmente en contextos vulnerables. Al mismo tiempo, el estudio podría servir de base para nuevas investigaciones centradas en los efectos de la integración de las tecnologías en la formación de los centros escolares.

Las futuras líneas de investigación tienen que ir enfocadas, haciendo uso de instrumentos de medida contrastados (Cabero, Marín\& Castaño, 2015), a investigar con los docentes, las familias, en especial los alumnos, triangulando los datos de fuentes tanto cuantitativas como cualitativas.

\section{Referencias}

Álvarez, M. \& Bisquerra, R. (2012). Orientación educativa: modelos, áreas, estrategias y recursos. Madrid: Wolters Kluwer.

Armstrong, L. (2014). 2013- the Year of Ups and Downs for the MOOCs. Changing Higher Education. Recuperado de http://goo.gl/SqwGWn/

Arufe-Giráldez, V., Soidán, J. L., Furelos R. \& Patón, R. (2016). El póster virtual, una nueva propuesta para la difusión del conocimiento en congresos. Sportis: Revista TécnicoCientífica del Deporte Escolar, Educación Física y Psicomotricidad, 2(3), 456-473.

Bernal, C. \&Rodríguez, A. (2007). La presentación de las prácticas docentes como vía para la integración curricular de los recursos digitales y el desarrollo profesional. Comunicación y Pedagogía, 222, 6.

Cabero, J., Marín, V. \& Castaño, C. (2015). Validación de la aplicación del modelo TPACK para la formación del profesorado en TIC. @tic. Revista d'innovació educativa, (14), $13-22$.

Carneiro, R. Toscano, J. C. \&Díaz, T. (2011). Los desafíos de las TIC para el cambio educativo. Madrid: Fundación Santillana.

Cohen, J. (1988) (2. ${ }^{a}$ ed.). Statistical Power Analysis for the Behavioral Sciences. New York: Academic Press.

Escudero, J. M., González, M. T. \& Rodríguez, M. J. (2018). Los contenidos de la formación continuada del profesorado: ¿Qué docentes se están formando? Educación XX1, 21 (1), 157-180.

García, F. \& Seoane, A. (2015). Una revisión actualizada del concepto de eLearning. Décimo Aniversario. Education In The Knowledge Society (EKS), 16(1), 119-144.

Gil, J. \& Martínez, J. (2017). El empoderamiento del alumnado en los sMOOC. Revista Complutense De Educación, 29(1), 43-60.

Gordon, D. (2003). Better Teaching and Leaning in Digital Classroom. Cambridge: Harvard Education Press.

Ley Orgánica 2/2006, de 3 de mayo, de Educación, (BOE, n 106 de 4 de mayo de 2006).

Marín, Verónica\& Llorente, María del Carmen (2015). Del e-Learning al e-PLE: renovando viejos modelos de enseñanza. Campus Virtuales, 2(2), 120-128.

Marín, V., Ramírez, A. \& Sampedro, B. (2011). Moodle y estudiantes universitarios. Dos nuevas realidades del EEES. Profesorado, Revista de currículum y formación del profesorado. 15(1), 109-120. Recuperado de: http://hdl.handle.net/10481/15362. 
Mengual, S. , Lloret, C. \& Roig, R. (2015). Validación del Cuestionario de evaluación de la calidad de cursos virtuales adaptado a MOOC. RIED. Revista lberoamericana de Educación a Distancia, 18 (2), 145-169.

Morales, A. B. (2010). La acción tutorial en Educación. Hekademos: Revista educativa digital, (7), 95-114. Recuperado de http://www.hekademos.com/hekademos/media/articulos/07/HEKADEMOS_N7.pdf.

Pérez, A. \& Rodríguez, M. J. (2016). Evaluación de las competencias digitales autopercibidas del profesorado de educación primaria en Castilla y León. Revista de Investigación Educativa,34(2), 399-415.

Roblizo, M. \& Cózar, R. (2015). Usos y competencias en TIC en los futuros maestros de educación infantil y primaria: hacia una alfabetización tecnológica real para docentes.Pixel-Bit. Revista de Medios y Educación, (47), 23-39.

Rodríguez, S. \& Romero, M. (2015). La función tutorial en Educación Infantil y Primaria: desempeño profesional del profesorado. Revista Electrónica Interuniversitaria de Formación del Profesorado, 18 (2), 43-56.

Royo, Y., Pérez, C., Almela, A., Pons, R., Herranz, C., Olivares, M. D., Montoya, F., Centenero, M. D, Martínez, J. F., Vivancos, R. \& Ochoa, Y. (2002). La Acción Tutorial en Primaria. Servicio de Ordenación Administrativa y Publicaciones. Consejería de Educación y Cultura. Región de Murcia.

Silva, J., Gros, B., Garrido, J. M. \& Rodríguez, J. (2006). Estándares en tecnologías de la información y la comunicación para la formación inicial docente: situación actual y el caso chileno. Revista Iberoamericana de Educación, 38(3), 1-17.

Solano, G. \& Espinoza, J. (2017). Formación de docentes para la creación de cursos virtuales en la enseñanza del español como segunda lengua. Comunicación, 26(1), 42-56.

Tello, J. \& Aguaded, J. I. (2009). Desarrollo profesional docente ante los nuevos retos de las tecnologías de la información y la comunicación en los centros educativos. Revista de Medios y Educación, (34), 31-47.

Torres, D. \& Gago, D. (2014). Los MOOCs y su papel en la creación de comunidades de aprendizaje y participación. RIED. Revista Iberoamericana de Educación a Distancia, 17(1), $13-34$. 